\title{
Defending Underdetermination or Why the Historical Perspective Makes a Difference
}

Wolfgang Pietsch, Chair for Philosophy of Science, Technische Universität München, Germany (pietsch@cvl-a.tum.de)

\begin{abstract}
:
The old antagonism between the Quinean and the Duhemian view on underdetermination is reexamined. In this respect, two theses will be defended. First, it is argued that the main differences between Quine's and Duhem's versions of underdetermination derive from a different attitude towards the history of science. While Quine considered underdetermination from an ahistorical, a logical point of view, Duhem approached it as a distinguished historian of physics. On this basis, a logical and a historical version of the underdetermination thesis can be distinguished. The second thesis of the article is that the main objections against underdetermination are fatal only to the logical rendering. Taken together, the two theses constitute a defence of underdetermination.
\end{abstract}

\section{Introduction}

It is revealing to compare W.V.O. Quine's "Two dogmas of empiricism", the locus classicus for his underdetermination thesis, with the relevant passages about underdetermination in Pierre Duhem's "The Aim and Structure of Physical Theory". While Duhem's book is filled with examples from the history of physics, in Quine's text historical references are almost completely lacking. While Duhem, the acclaimed historian of physics, was practising philosophy of science from a genuinely historical perspective, Quine approached underdetermination from an ahistorical, a logical point of view.

In Section Two, it will be argued that the respective attitudes towards the history of science lie at the origin of the most important differences between these two main versions of the underdetermination thesis. Also, the stance on history can serve as a guideline for detecting further differences. All this allows us to refer to Quine's perspective as the logical rendering and to Duhem's as the historical rendering of underdetermination.

In Section Three, we evaluate both versions of underdetermination with respect to the main objections that have been brought forward in the literature. Remarkably, they turn out fatal only to the logical rendering. This argument in defence of the historical version of underdetermination provides further justification for the recent interest in transient underdetermination, largely due to the significant and insightful work of Kyle Stanford (2001, 2006, 2009). As Stanford readily acknowledges, transient underdetermination owes much to a Duhemian spirit (2006, Ch. 2) and therefore to the historical rendering of underdetermination.

In the last Section we conclude with some positive remarks about the role of underdetermination in the scientific enterprise. Essentially, Duhem's version of the underdetermination thesis is a necessary and indispensable tool for the historical reconstruction of science and it is an epistemic reminder that science should be pluralistic and always tolerant towards alternative approaches. Much in the spirit of Carrier (2009), a shift in focus is advocated away from seeing underdetermination principally as an argument in the realism-antirealism debate towards examining the significance of underdetermination for scientific method.

\section{The logical and the historical view on underdetermination}

Quine once claimed that Duhem never endorsed underdetermination at all (1975, 313). In a literal sense, this is correct given that Quine defines underdetermination with respect to all possible evidence while Duhem always considers evidential situations in an actual historical context. However, from a larger perspective Quine's insistence is profoundly misleading. Quine crucially overlooks that the underdetermination thesis constitutes a closely-knit web with other Duhemian 
ideas, especially the theory-ladenness of observation, confirmational holism, and the impossibility of an experimentum crucis - if underdetermination is understood simply in the sense that theory is not uniquely determined by evidence. In this formulation, which will be used from here on, the underdetermination thesis is compatible with both Duhem's and Quine's views. Still, there are notable differences, to which we will turn now.

Several scholars have compared Quine's and Duhem's rendering of the underdetermination thesis (Vuillemin 1978, Ariew 1984, Quine 1986, Gillies 1993) ${ }^{1}$. But all these authors fail to come up with a general rationale driving the different viewpoints of Duhem and Quine. In this section the claim will be defended that all the main differences between Duhem's and Quine's versions of underdetermination derive from their respective stance towards the history of science. Quine approaches underdetermination from an ahistorical, a logical point of view. His 'in-principle' viewpoint determines only the boundaries that pure deductive logic imposes on scientific theorizing. Such logical restrictions fall short of providing a sufficient basis for Duhem's interest in reconstructing episodes from the history of physics.

The key difference noted and agreed upon by all scholars is that Duhem imposes careful limitations on the underdetermination thesis while Quine refrains from doing so by stating claims that are all largely tenable only "in legalistic principle" (Quine 1986, 619). In more detail, such limitations include (Gillies 1993, 313-314): (i) Duhem restricts underdetermination to a specific type of statements, namely theoretical statements in abstract sciences like physics ${ }^{2}$. Quine denies any such distinction framing his discussion in an outright denial of the analytic/synthetic distinction per se. (ii) Relatedly, the scope of the thesis is specified differently by Duhem and Quine. Quine thought it to concern the whole of science, from logic and mathematics to the almost purely phenomenological sciences. By contrast, Duhem claims that phenomenological sciences like physiology are not affected by the ambiguities of underdetermination. (iii) Duhem makes reference to 'good sense' as an aid complementary to deductive logic, which will (at least in most cases) tell the physicist which theory to choose. Quine's account includes nothing of this sort.

It is quite obvious, why someone interested in the reconstruction of the history of physics cannot work with Quine's over-generalizations and in-principle statements. Of course, there is some legalistic truth to Quine's denial of the analytic-synthetic distinction, i.e. to his claim that all statements inseparably contain an analytic linguistic and a synthetic empirical component. But the working scientist and the historian of science have to assume a much more pragmatic attitude. If a scientific theory is to be useful, a distinction between 'pure' observation statements (e.g. 'The needle of my measuring device points to 10.'), abstract hypotheses in advanced theories and fully analytic statements like the principles of deductive logic is essential.

More often than not, we find general agreement from a practical point of view on the analytic or synthetic nature of a scientific proposition. Historians and working scientists just have to accept such unanimity in the overwhelming majority of cases. However, in a certain transition region, the synthetic and analytic parts are indeed very hard to disentangle, for example when dealing with central hypotheses in abstract sciences. When we delve into the history of science, this transition region is often fiercely embattled lacking the consensus that we described above. E.g. if we look at fundamental axioms in physical theories, these cannot be purely analytic statements, since they clearly tell us something about the world. But they cannot be purely synthetic either, since universal validity cannot be based on experience alone. It is primarily in this transition region

\footnotetext{
1 Not all these sources list differences between Duhem's and Quine's versions of underdetermination, some only compare Duhem and Quine with respect to their holism. But since the concepts of underdetermination and holism are intricately connected, such differences immediately apply to the underdetermination thesis as well. Among these authors, Veuillemin is the only one to notice that Duhem renders underdetermination as a historical thesis with Quine being "as indifferent as Americans often are concerning history" (Vuillemin 1978, 598). However, Veuillemin also misses the overall importance of this difference in perspective.

2 Duhem has been largely misunderstood in his alleged restriction of underdetermination to physics. Plausibly, Duhem considered himself an expert only in the methodology of physics and thus did not dare to judge the possible extension of these ideas to other sciences: "But if it is good to notice the analogies between our diverse scientific methods, it is on condition that we do not forget the differences separating them." (Duhem 1990, 187)
} 
between the synthetic and the analytic, where Duhem's interconnected theses about theoryladenness of observation, holism, impossibility of an experimentum crucis and underdetermination become relevant.

Relatedly, neither the historian of science nor the working scientist will insist on the interconnectedness of all knowledge in the way Quine stresses it. For Quine, "[t]he totality of our so-called knowledge or beliefs, from the most casual matters of geography and history to the profoundest laws of atomic physics or even of pure mathematics and logic, is a man-made fabric which impinges on experience only along the edges" (Quine 1951, 42). For Quine, underdetermination will never concern only clearly delineated areas of scientific knowledge but will always affect the whole of science. By contrast, Duhem limits the scope of underdetermination to the net of hypotheses that make up the core of abstract theories. For the working scientist as well as for the historian of science, Quine's viewpoint is untenable. Surely, hypotheses within physics can be discussed without considering physiology, genetics, or the trivial problems troubling our everyday life. Fortunately, a particle physicist carrying out experiments at CERN need not be aware of the fact that you are reading this article now. Of course, Quine is right that in principle all knowledge is connected through logic and mathematics. In extreme situations unexpected connections might even become relevant, but in general Quine's extreme holism will make any scientific endeavour impossible. If underdetermination is to play a role for scientific method, then only on the level of clearly delineated groups of hypotheses within abstract scientific theories.

Finally, once the historian of science accepts the possibility of underdetermination, she needs an account of 'good sense' to make sense of historical episodes where scientists eventually settled on one of the rivalling accounts in spite of the in-principle impossibility of an experimentum crucis. Essentially, a historian who accepts underdetermination faces the choice between two options: either the decision-making of the scientists was fully irrational and therefore determined largely by social factors or the decision-making relied on norms and rules that fall short of being fully rational while nevertheless being better justified than purely subjective arbitrariness. It is the second option that Duhem chooses with his theory of good sense: "But these reasons of good sense do not impose themselves with the same implacable rigor that the prescriptions of logic do. There is something vague and uncertain about them; they do not reveal themselves with the same degree of clarity to all minds." (Duhem 1906/1954, 217) Duhem's theory of good sense and his nuanced account of scientific method show the sensibility of a distinguished historian to the diversity of influences shaping scientific evolution, in stark contrast to the coarseness of Quine's approach which comes close to denying any distinction that cannot be drawn with absolute logical rigour as well as any norms or rules that do not satisfy the strict rationality criteria of logical thinking. ${ }^{3}$

Once it has become clear, that the different stance on the history of science lies at the origin of the differences between Duhem's and Quine's versions of underdetermination, this insight can serve as a guiding principle to detect further distinctions. Remarkably, this principle reveals a number of further crucial differences, which have not been mentioned in any of the references cited at the beginning of this section.

(iv) Duhem (1906/1954) discusses underdetermination in relation to the evidence in an actual historical situation, while Quine (1975) relates underdetermination to all possible evidence. The focus shifts from actually observed events to events that are observable in principle. Obviously, a historian of science or a working scientist will always address a scientific problem in the context of a specific historical situation. Only the logician has the privilege to abstract from the historical context with respect to evidence and enter the realm of possible evidence. To the working scientist, the idea of possible evidence is largely meaningless. In addition, even if agreement between two theories with respect to all possible evidence could be established, this would immediately render the case uninteresting for the working scientist. Arguably, a working scientist is much more interested in theories that largely agree with respect to the available evidence but delineate different

\footnotetext{
Quine admits to some pragmatic criteria for theory choice, for example that statements in the periphery are less costly to change than those pertaining to the centre of the web of knowledge. But these occasional remarks fall far short of the sophistication of Duhem's account.
} 
research agendas by making different predictions.

(v) Relatedly, when dealing with actual episodes of underdetermination from the history of science, rival theories generally fail to be fully equivalent even with respect to past evidence. While having a large overlap, the domains of application will be somewhat different. Also, the competing accounts will deviate from each other in the reconstruction of available evidence, both in numbers and exactness. A good way of thinking about such rival theories is that they are potentially equivalent, i.e. with sufficient ingenuity they can generally be made to agree with each other. In summary, actual cases of underdetermination concern competing accounts, where different ontologies provide the starting point for different future research programs. A situation of underdetermination does not require complete empirical equivalence, not even with regard to past evidence. Rather, underdetermination arises in the moment, when a choice between both frameworks cannot be made by relying on the usual criteria of empirical adequacy and of epistemic virtues.

(vi) The ahistorical nature of Quine's rendering of underdetermination is well illustrated by his choice of examples. They are mostly constructed from contemporary theories or even from nonscientific parts of knowledge, while Duhem searches the history of science for relevant episodes. Quine, the logician and linguist, starts an unfortunate chain of constructed and algorithmic examples involving for example redefinition of terms, reformulation of logic, hallucinations and the like (1951, 36, 42-46). Much confusion has resulted from not properly distinguishing examples of the historical kind from the logical kind, for example when actual historical episodes like the underdetermination of matrix and wave mechanics are discussed in terms of empirical equivalence. This insight also points us to a possible explanation for the alleged lack of examples for underdetermination in the history, which has often been cited by opponents of the underdetermination thesis (e.g. Norton 2008, 25). If you search the history of science with empirical equivalence in mind, you will certainly miss the most pertinent examples of underdetermination like the episode from electrodynamics that will be presented in the next section.

(vii) Last not least, there is a difference in aim. While Quine's version of the thesis has mostly found its place as an argument in linguistic debates concerning the analytic-synthetic distinction (1951) or the indeterminacy of translation (1970) as well as in the realism/antirealism debate, Duhem's version with its historical outlook is an important tool both for the historian and the working scientist. Duhem's version finds its place naturally in scientific method. If we want underdetermination to be relevant to the mature sciences like physics, chemistry or biology, then we have no other choice but to accept Duhem's viewpoint.

\section{Defending the historical version of underdetermination}

In this section we will argue that the main criticisms of the underdetermination thesis are fatal only to the logical version of the thesis. The two most important arguments against underdetermination respectively counter the two principal strategies that have been employed in defence of underdetermination. First, the identical rivals objection counters what John Norton calls the inductive argument for underdetermination, which relies on citing examples (2008, 24). Second, the objection from an impoverished account of confirmation attempts to undermine the holistic argument for underdetermination. Let us discuss these in turn.

Following the inductive strategy, a proponent of underdetermination should ideally produce a long list of poignant examples showing that underdetermination is a persistent phenomenon in scientific development. By contrast, opponents of underdetermination could engage in a piece-meal attack showing that each of these examples is either trivial, misconstrued, or for other reasons invalid. Space is too limited here to address any of the debates concerning specific historical episodes in detail. Let us just remark that sometimes historical examples are supposedly refuted on the basis that the theories were not really empirically equivalent. It should be clear by now that this mixes up the logical and the historical versions of underdetermination.

There exist also general strategies against the inductive justification of underdetermination. 
The most important one has been called the identical rivals objection, which argues that alleged examples of underdetermination always concern only variant formulations of the same theory (Quine 1975, Magnus 2003, Norton 2008, Frost-Arnold and Magnus 2009). A clever version was proposed by John Norton, who in an ingenious single sweep attempts to invalidate a large class of examples: „The very fact that observational equivalence can be demonstrated by arguments brief enough to be included in a journal article means that we cannot preclude the possibility that the theories are merely variant formulations of the same theory." $(2008,17)$

Clearly, this objection concerns only the Quinean type of constructed examples, for which a short algorithm can be given within the scope of a journal article-involving redefinitions of terms, hallucinations, Cartesian demons, brains in the vat etc. The Duhemian ones, naturally grown in the history of science, remain unrefuted. Duhem's examples are those that were developed over large periods of time and by a considerable number of scientists. In these cases, any equivalence-proof is an immensely intricate affair, never to be accomplished in a single journal article. Too often, philosophers arguing for underdetermination have given in to the temptation to present clear-cut algorithmic examples rather than engaging in detailed historical studies (Quine 1951, Kukla 1996). Stanford has rightly pointed out that this strategy is self-defeating and has called it a "devil's bargain” (Stanford 2001): „In retrospect, perhaps it should not surprise us that philosophers' algorithms cannot make short work of the daunting task of generating alternative hypotheses that are both scientifically serious and genuinely distinct from existing competitors, for this is precisely the sort of difficult conceptual achievement that demands the sustained efforts of real scientists over years, decades, and even careers." (Stanford 2006, 15) True examples of underdetermination can only come from the history of science, in rare exceptions also from contemporary science.

Let me draw your attention to an excellent example of underdetermination from the history of electrodynamics, which has not been mentioned at all in the recent literature - at least to my knowledge. In the second half of the $19^{\text {th }}$ century, there was agreement among some leading figures, including James Clerk Maxwell and William Thomson, that the relation between the continental action-at-a-distance approach and the British field theoretic approach constitutes a situation of underdetermination (for more details cf. Pietsch 2010). Maxwell writes: "[I]t is exceedingly important that two methods should be compared, both of which have succeeded in explaining the principal electromagnetic phenomena, and both of which have attempted to explain the propagation of light as an electromagnetic phenomenon, and have actually calculated its velocity, while at the same time the fundamental conceptions of what actually takes place, as well as most of the secondary conceptions of the quantities concerned, are radically different." (Maxwell 1873, x) This is an exemplary assessment of a situation of historical underdetermination in one of the most prominent places of Maxwell's work, the introduction to the Treatise on Electricity and Magnetism.

It is immediately obvious that we are not dealing with identical rivals here since both programs are worlds apart in terms of ontology and methodology. Also, both of them have contributed crucially to the development of electrodynamics. Furthermore, while the two programs can certainly be connected via elaborate bridge laws there does not exist any straightforward algorithm that turns one theory into the other.

In this context we can address another objection that has been voiced in connection with underdetermination, which might be called the objection from scientific import. What if anything, so a working scientist could ask, does science gain from observationally equivalent theories? Why not take the simplest approach and forget about the others? Well granted, the constructed examples are largely uninteresting for science and scientific methodology. But the historical examples are not observationally equivalent in the abstract algorithmic way of logical underdetermination. Rather, the rivalling theories in historical underdetermination provide the researchers with widely different perspectives on the world. Only in retrospect, theories can often be made to fit all data. But this does not imply any strict equivalence from the outset. Once you try to press actual 'living' science in a logical corset, much is lost.

A proponent of logical underdetermination might object that underdetermination is about theories that rely on different metaphysics in terms of ontology, mathematical structure, etc. while 
being nevertheless observationally equivalent. The reply of the proponent of the historical view would point out that this misconstrues the role of metaphysics in science. The metaphysical foundations of a theory are never independent of the empirical research to be undertaken. Metaphysics offers a necessary and indispensable guideline for the inductive business of science: which experiments to do, which theoretical problems to tackle next. Underdetermination is really about equally strong theories with different metaphysics, that provide the scientist with different instructions what to do next and what to expect from nature.

Let us quickly illustrate this by means of the mentioned example from electrodynamics. The field view populates the world with a wholly different ontology in comparison with the particles of the action-at-a-distance view. These different ontologies provide a completely different perspective on the world whose influence can be traced in the different merits of the theories. In short, while the focus in the field view is on the space in between, the focus in action at a distance is on matter interacting across a distance. For example, the action-at-a-distance paradigm has historically contributed to electrodynamics the formulation of the fundamental laws of electro- and magnetostatics modelled on Newtonian action at a distance (Coulomb), and the unification of electrostatic and electrodynamic interaction (Weber). On the other hand, the field view has contributed the theories of dielectrics and diamagnetism, i.e. of electromagnetic media (Faraday), and the unification of optics and electrodynamics (Maxwell).

This perspective dissolves another objection against underdetermination, which was raised by Larry Laudan and Jarrett Leplin. According to them, any assessment of "empirical equivalence is both contextual and defeasible" $(1991,454)$. This correct remark casts again much doubt on the logical formulation of underdetermination with its insistence on logical equivalence. By contrast, the remark only underscores the historical view on underdetermination with its focus on potential equivalence - a notion which is historically relative and also depends on the willingness and ingenuity of the protagonists to make different frameworks fit the data.

Let us finally address the second major objection against underdetermination mentioned at the beginning of this section. It attacks the holistic argument for underdetermination. According to this maybe most common objection, the underdetermination thesis relies on an impoverished account of confirmation ${ }^{4}$. And indeed, both Duhem and Quine largely employ a hypotheticodeductive methodology to argue for underdetermination, largely ignoring the wide variety of inductive methods. ${ }^{5}$ In short, their argument goes as follows. Because in every experimental test of abstract hypotheses a plethora of auxiliary hypotheses must be employed, no observation can ever falsify a specific abstract hypothesis. In principle then, we can always stick to certain abstract hypotheses, no matter what the evidence is. Therefore different scientists, who stick to mutually contradictory ontological hypotheses, can never be proven wrong in principle. There is little doubt that this argument for underdetermination with its emphasis on falsification relies crucially on a hypothetico-deductive framework. Opponents of underdetermination then claim, that the argument fails to work if we add inductive methods. ${ }^{6}$ Sometimes (but not too often) a detailed analysis is provided for this claim and it is shown how specific inductive techniques actually resolve cases of underdetermination (e.g. in Norton 2008, 29-32). Given the limited scope of this paper, we have to leave a reply to these arguments for another occasion.

Fortunately from the historical perspective, we need not counter any of these specific claims. While it is indeed plausible that the constructed and algorithmic examples can be invalidated by means of inductive methods, this is obviously not true for the historical examples. Every reproach, that an impoverished scientific method was used in these situations, would immediately carry over to the respective scientists themselves. In the example from electrodynamics, the charge would

\footnotetext{
An excellent overview and introduction to this objection can be found in (Norton 2008, 26-32).

Duhem does include a thorough critique of inductive methods (1906/1954, Part II Ch. VI).

Some have equated Duhemian 'good sense' with inductive methods. But Duhem explicitly insisted on good sense not being rationally reconstructible (cp. the quote in Section Two). When Duhem claims that good sense often resolves episodes of underdetermination, this is decidedly not equivalent with claiming that induction solves the problem.
} 
immediately be referred to Maxwell and Thomson. It is clearly absurd to claim, that these men, who are among the best physicists in the history of science, relied on an impoverished methodology for physics.

So far, we have examined a variety of objections against underdetermination and have found that while several are indeed fatal to Quine's logical version they generally fail even to be relevant to Duhem's version. The simple reason is that the majority of them rely on the doubtful concept of empirical or observational equivalence with respect to possible evidence. However, there is another objection which undermines the holistic argument for underdetermination and which is indeed relevant to the historical view on underdetermination. It seems to have been first formulated by Grünbaum: „Duhem cannot guarantee on any general logical grounds the deducibility of $\mathrm{O}$ [empirical findings] from an explanans constituted by the conjunction of $\mathrm{H}$ [empirical hypothesis] and some revised non-trivial version R of A [auxiliary assumptions].“ (1960, 75) Granted, but this asks too much from Duhem, anyhow. There are never any logical guarantees for the existence of scientific theories. In the end, we are faced with a stalemate between the opponent and the proponent of underdetermination since the non-existence of alternatives cannot be logically proven either. Maybe then, we should give underdetermination the benefit of the doubt. After all, if it cannot be ruled out, then we should be methodologically prepared for it.

\section{Conclusion}

Two claims were defended in this article. First, all differences between the Quinean and the Duhemian rendering of underdetermination can be made plausible from a difference in perspective: Duhem from a historical, Quine from a logical point of view. Second, the neglect of the historical perspective has been detrimental: The fatal objections against underdetermination concern only the Quinean rendering. Much remains to be done. In particular, we need to have a detailed look at specific examples of underdetermination from the history of science. Also, further research is necessary on the exact methodological implications of underdetermination in the abstract sciences. Whatever the results of these studies may be, Duhem's rendering is useful as a tool for understanding the historical development of science and stands as an epistemological reminder that science should not be dogmatic but tolerant to alternative perspectives.

\section{Acknowledgements}

I am grateful to Mauricio Suárez for helpful comments and suggestions as well as to audiences at EPSA09 in Amsterdam and at \&HPS2 in Notre Dame.

\section{References}

Ariew, Roger. 1984. The Duhem Thesis. British Journal for the Philosophy of Science 35: 313-325. Duhem, Pierre. 1906/1954. The Aim and Structure of Physical Theory. Princeton, NJ: Princeton University Press. Translation of La Théorie physique. Son objet et sa structure. Paris: Chevalier \& Rivière.

Duhem, Pierre. 1990. Logical Examination of Physical Theory. Synthese 83: 183-188.

Carrier, Martin. 2009. Underdetermination as an Epistemological Test Tube: Expounding Hidden Values of the Scientific Community. Synthese. doi: 10.1007/s 11229-009-9597-6.

Frost-Arnold, Greg and P. D. Magnus. 2009. The Identical Rivals Response to Underdetermination. PhilSci archive. http://philsci-archive.pitt.edu/archive/00003390/. Accessed 15 February 2010.

Gillies, Donald. 1993. Philosophy of Science in the Twentieth Century. Oxford: Blackwell. Excerpt reprinted in Philosophy of Science. The Central Issues, ed. M. Curd and J. A. Cover, 302319. New York: Norton.

Grünbaum, Adolf. 1960. The Duhemian Argument. Philosophy of Science 27 (1): 75-87. 
Kukla, André. 1996. Does Every Theory Have Empirically Equivalent Rivals? Erkenntnis 44: 137166.

Laudan, Laurens. 1965. Discussion: Grünbaum on "The Duhemian Argument". Philosophy of Science 32 (3/4): 295-299.

Laudan, Larry. 1990. Demystifying Underdetermination. In Scientific Theories, ed. C. Wade Savage, 267-297. Minneapolis: University of Minnesota Press.

Laudan, Larry and Jarrett Leplin. 1991. Empirical Equivalence and Unterdetermination. The Journal of Philosophy 88 (9): 449-472.

Magnus, P. D. 2003. Underdetermination and the Problem of Identical Rivals. Philosophy of Science 70: 1256-1264.

Maxwell, James C. 1873. A Treatise on Electricity and Magnetism. Oxford: Clarendon Press.

Norton, John D. 2008. Must Evidence Underdetermine Theory? In The Challenge of the Social and the Pressure of Practice: Science and Values Revisited, ed. M. Carrier, D. Howard, and J. Kourany, 17-44. Pittsburgh: University of Pittsburgh Press.

Pietsch, Wolfgang. 2010. On Conceptual Issues in Classical Electrodynamics: Prospects and Problems of an Action-at-a-distance Interpretation. Studies in History and Philosophy of Modern Physics 41 (1): 67-77.

Quine, Willard V. O. 1951/1980. Two Dogmas of Empiricism. Philosophical Review 60: 20-43. Reprinted in From a Logical Point of View: 9 Logico-Philosophical Essays, 20-46. Cambridge, MA: Harvard University Press.

Quine, Willard V. O. 1975. On Empirically Equivalent Systems of the World. Erkenntnis 9: 313328.

Quine, Willard V. O. 1986. Reply to Jules Vuillemin. In The Philosophy of W. V. Quine, ed. Lewis E. Hahn and Paul A. Schilpp, 619-622. La Salle: Open Court.

Sklar, Lawrence. 1975. Methodological Conservatism. Philosophical Review 84: 384-400.

Stanford, P. Kyle. 2001. Refusing the Devil's Bargain: What Kind of Underdetermination Should we Take Seriously? Philosophy of Science 68: S1-S12.

Stanford, P. Kyle. 2006. Exceeding Our Grasp. Oxford: Oxford University Press.

Stanford, P. Kyle. 2009. Underdetermination of Scientific Theory. In Stanford Encyclopedia of Philosophy (Winter 2009 Edition), ed. Edward N. Zalta. http://plato.stanford.edu/archives/win2009/entries/scientific-underdetermination/.

Vuillemin, Jules. 1979. On Duhem's and Quine's Theses. Grazer philosophische Studien 9: 69-96. Reprinted in The Philosophy of W. V. Quine, ed. Lewis E. Hahn and Paul A. Schilpp, 595618. La Salle: Open Court. 